\title{
Abbreviations and acronyms of books, documents and periodicals
}

AIPLA Q.J.

AJIL

AJP

All E.R.

Am.J.Comp.L.

BIE

$\mathrm{CA} /$.

CA/PL . .

Case W. Res. J.

INT'L LAW

C.L.J.

CML REv.

ECR

E.L.J.

E.L.R.

E.L.REV.

EIPR

EuZ

EuZW

F.2d

F. Supp.

F.S.R.

GRUR

GRUR INT.

H.L. Cas.

HARV.INT'L.L.J.

HARV.L.R.
AIPLA Quarterly Journal (USA)

American Journal of International Law (USA)

Aktuelle Juristische Praxis $(\mathrm{CH})$

All England Law Reports (UK)

The American Journal of Comparative Law

(USA)

Bijblad bij de Industriële Eigendom (NL)

Document of the Administrative Council of the

European Patent Organisation

Document of the Committee on Patent Law of the European Patent Organisation

Case Western Reserve Journal of International

Law (USA)

Cambridge Law Journal (UK)

Common Market Law Review (NL)

European Court Reports (EU)

European Law Journal (IT/FR/UK)

European Law Reporter (LU/CH)

European Law Review (UK)

European Intellectual Property Review (UK)

Zeitschrift für Europarecht $(\mathrm{CH})$

Europäische Zeitschrift für Wirtschaftsrecht

(DE)

Federal Reporter (2nd ser.; USA)

Federal Reporter, Supplement (USA)

Fleet Street Reports (UK)

Gewerblicher Rechtsschutz und Urheberrecht (DE)

Gewerblicher Rechtsschutz und Urheberrecht Internationaler Teil (DE)

Clark's House of Lords Cases 1847-1866 (UK)

Harvard International Law Journal (USA)

Harvard Law Review (USA) 
xxii

ICLQ

IIC

I.L.Pr.

ICIP-ING.CONS.

IPQ

IPRax

J.D.I.

JIPLP

JWIP

JZ

LQR

Mitt.

NJ

NJW

ÖB1

O.J. EPO

O.J. EC

O.J. EU

P.I.P.D.

p.i.

propr. industr.

RABELSZ

R.D.P.I.

RIW

RPC

RLDA

SJZ

SMI
European patent law

International and Comparative Law Quarterly

(UK)

International Review of Industrial Property and

Copyright Law (DE)

International Litigation Procedure (UK)

L'Ingenieur Conseil - Intellectual Property (BE)

Intellectual Property Quarterly (UK)

Praxis des Internationalen Privat- und

Verfahrensrechts (DE)

Journal du Droit International (FR)

Journal of Intellectual Property Law \& Practice (UK)

The Journal of World Intellectual Property $(\mathrm{CH} /$ UK)

Juristen-Zeitung (DE)

The Law Quarterly Review (UK)

Mitteilungen der deutschen Patentanwälte

(DE)

Nederlandse Jurisprudentie (NL)

Neue Juristische Wochenschrift (DE)

Österreichische Blätter für gewerblichen

Rechtsschutz und Urheberrecht (AT)

Official Journal of the European Patent Office (EPO)

Official Journal of the European Community (EC)

Official Journal of the European Union (EU)

Propriété Industrielle Bulletin Documentaire (FR)

Propriétés intellectuelles (FR)

Propriété industrielle (FR)

Rabels Zeitschrift für ausländisches und internationales Privatrecht (DE)

Revue du Droit de la Propriété Intellectuelle (FR)

Recht der Internationalen Wirtschaft (DE)

Reports on Patent, Design and Trademark cases

(UK)

Revue Lamy, Droit des affaires (FR)

Schweizerische Juristenzeitung $(\mathrm{CH})$

Schweizerische Mitteilungen über

Immaterialgüterrecht $(\mathrm{CH})$ 
sic!

Zeitschrift für Immaterialgüter-, Informationsund Wettbewerbsrecht $(\mathrm{CH})$

U.Pa.L.Rev. University of Pennsylvania Law Review (USA)

U.S.P.Q. United States Patent Law Quarterly (USA)

VA.J.INT'L.L.

Virginia Journal of International Law (USA)

VAND.J.TranSNAT'L.L. Vanderbilt Journal of Transnational Law (USA)

VPP-Rundbrief

Rundbrief der Vereinigung von Fachleuten auf

dem Gebiet des gewerblichen Rechtsschutzes

(DE)

Wash.U.J.L. \& PoL'Y. Washington University Journal of Law \& Policy (USA)

WIPO/ACE/ ... D Document of the WIPO Advisory Committee on Enforcement

WPL/../. $\quad$ Document of the Working Party on Litigation

WPL/SUB ../.. $\quad$ Document of the Sub-group of the Working

Party on Litigation (European Patent

Organisation)

WIPR

World Intellectual Property Report (USA/UK)

Y.B.E.L.

Yearbook of European Law (UK)

ZBJV

Zeitschrift des Bernischen Juristenvereins $(\mathrm{CH})$

ZEuP

Zeitschrift für europäisches Privatrecht (DE)

ZfRV

Zeitschrift für Rechtsvergleichung (AT)

ZSR

Zeitschrift für schweizerisches Recht $(\mathrm{CH})$

ZZP

Zeitschrift für Zivilprozess (DE)

ZZPInt

Zeitschrift für Zivilprozess International (DE) 
Stefan Luginbuehl - 9780857933218 